Georgian Mathematical Journal

1(1994), No. 1, 9-29

\title{
CRITERIA OF GENERAL WEAK TYPE INEQUALITIES FOR INTEGRAL TRANSFORMS WITH POSITIVE KERNELS
}

\author{
I. GENEBASHVILI, A. GOGATISHVILI, AND V. KOKILASHVILI
}

Abstract. Necessary and sufficient conditions are derived in order that an inequality of the form

$$
\begin{gathered}
\varphi(\lambda) \theta(\beta\{(x, t) \in X \times[0, \infty): \mathcal{K}(f d \nu)(x, t)>\lambda\}) \leq \\
\leq c \int_{X} \psi\left(\frac{f(x)}{\eta(\lambda)}\right) \sigma(x) d \nu(x)
\end{gathered}
$$

be fulfilled for some positive $c$ independent of $\lambda$ and a $\nu$-measurable nonnegative function $f: X \rightarrow \mathbf{R}^{1}$, where

$$
\mathcal{K}(f d \nu)(x, t)=\int_{X} f(y) k(x, y, t) d \nu(y), t \geq 0,
$$

$k: X \times X \times[0, \infty) \rightarrow \mathbf{R}^{1}$ is a nonnegative measurable kernel, $(X, d, \mu)$ is a homogeneous type space, $\varphi \eta$ and $\psi$ are quasiconvex functions, $\psi \in \Delta_{2}$, and $t^{-\alpha} \theta(t)$ is a decreasing function for some $\alpha, 0<\alpha<1$.

A similar problem was solved in Lorentz spaces with weights.

\section{INTRODUCTION}

This paper presents a characterization of weight functions and kernels for which we have general weight weak type inequalities for integral transforms of the form

$$
\mathcal{K}(f d \nu)(x, t)=\int_{X} f(y) k(x, y, t) d \nu(y)
$$

where $X$ is a homogeneous type space, and $k: X \times X \times[0, \infty) \rightarrow \mathbf{R}^{1}$ a nonnegative measurable kernel.

The homogeneous type space $(X, d, \mu)$ is a space with measure $\mu$ such that the class of compactly supported continuous functions is dense in the space $L^{1}(X, \mu)$. Moreover, it is also assumed that there is a nonnegative real-valued function $d: X \times X \rightarrow \mathbf{R}^{1}$ satisfying the following conditions:

1991 Mathematics Subject Classification. 42B25,26D15,46E30. 
(i) $d(x, x)=0$ for all $x \in X$;

(ii) $d(x, y)>0$ for all $x \neq y$ in $X$;

(iii) There is a constant $a_{0}$ such that $d(x, y) \leq a_{0} d(y, x)$ for all $x, y$ in $X$;

(iv) There is a constant $a_{1}$ such that $d(x, y) \leq a_{1}(d(x, z)+d(z, y))$ for all $x, y, z$ in $X$

(v) For each neighborhood $V$ of $x$ in $X$ there is an $r>0$ such that the ball $B(x, r)=\{y \in X: d(x, y)<r\}$ is contained in $V$;

(vi) The balls $B(x, r)$ are measurable for all $x$ and $r>0$;

(vii) There is a constant $b$ such that $\mu B(x, 2 r) \leq b \mu B(x, r)$ for all $x \in X$ and $r>0$ (see [1], p. 2).

In the sequel $\hat{B}(x, r)$ will denote the set $B(x, r) \times[0,2 r)$ for $r>0$ and the one-point set $\{x\}$ for $r=0$. The set $B(x, 0)$ will be assumed to be empty. $\beta$ will be a measure defined on the product of $\sigma$-algebras generated by balls in $X$ and by intervals from $[0, \infty)$.

Let $\varphi, \psi$, and $\eta$ be nonnegative nondecreasing functions on $[0, \infty)$. For our further discussion we will also need the following basic definitions of quasiconvex functions. We call $\omega$ a Young function if it is a nonnegative increasing convex function on $[0, \infty)$ with $\omega(0)=0, \omega(\infty)=\infty$ and not identically zero or $\infty$ on $(0, \infty)$; it may have a jump up to $\infty$ at some point $t>0$, but in that case it should be left continuous at $t$ (see [2]).

The function $\psi$ is called quasiconvex if there exist a Young function $\omega$ and a constant $c>1$ such that

$$
\omega(t) \leq \psi(t) \leq \omega(c t), \quad t \geq 0 .
$$

Clearly, $\psi(0)=0$, and for $s \leq t$ we have $\psi(s) \leq \psi(c t)$. To the quasiconvex function $\psi$ we can put into correspondence its complementary function $\tilde{\psi}$ defined by $\tilde{\psi}(t)=\sup _{s \geq 0}(s t-\psi(s))$.

The subadditivity of the supremum easily implies that $\tilde{\psi}$ is always a Young function and $(\tilde{\psi})^{\sim} \leq \psi$. The equality holds if $\psi$ is itself a Young function.

If $\psi_{1} \leq \psi_{2}$, then $\tilde{\psi}_{2} \leq \tilde{\psi}_{1}$, and if $\psi_{1}(t)=a \psi(b t)$, then $\tilde{\psi}_{1}(t)=a \tilde{\psi}\left(\frac{t}{a b}\right)$. Hence from (1.2) we have

$$
\tilde{\omega}\left(\frac{t}{c}\right) \leq \tilde{\psi}(t) \leq \tilde{\omega}(t) .
$$

Now from the definition of $\tilde{\psi}$ we obtain Young's inequality

$$
s t \leq \psi(s)+\tilde{\psi}(t), \quad s, t \geq 0 .
$$

It should be noted that unlike $\psi$ the function $\tilde{\psi}$ may jump to $\infty$ at some point $t>0$. For example, if $\psi(t)=t$, then $\tilde{\psi}(t)=\infty \cdot \chi_{(1, \infty)}(t)$. Throughout the paper we take $0 \cdot \infty$ to be zero. 
We use the convention that $c$ denotes the absolute constant which may change from line to line.

The function $\psi$ satisfies the (global) $\Delta_{2}$ condition $\left(\psi \in \Delta_{2}\right)$ if there exists $c>0$ such that $\psi(2 t) \leq c \psi(t), t>0$.

Some properties of quasiconvex functions and also of functions satisfying the $\Delta_{2}$ condition will be presented in Section 2 .

Now we are ready to formulate the main results of this paper.

In the sequel $\theta$ will always be a positive nondecreasing function.

Theorem 1.1. Let $k: X \times X \times[0, \infty) \rightarrow \mathbf{R}^{1}$ be any measurable nonnegative kernel, let $\psi \in \Delta_{2}$, and let the function $t^{-\alpha} \theta(t)$ decrease for some $\alpha \in(0,1)$. Let, further, $\nu$ be a finite measure on $X$, and $\sigma: X \rightarrow \mathbf{R}^{1}$ be an almost everywhere positive function which is locally summable with respect to measure $\nu$.

Assume that there exist positive constants $\varepsilon$ and $c_{1}$ such that

$$
\begin{gathered}
\int_{X \backslash B(a, r)} \tilde{\psi}\left(\varepsilon \frac{\varphi(s) \eta(s)}{s} \frac{\theta\left(\beta \hat{B}\left(a, N_{0}(2 r+t)\right)\right)}{\sigma(y)} k(a, y, t)\right) \sigma(y) d \nu(y) \leq \\
\leq c_{1} \varphi(s) \theta\left(\beta \hat{B}\left(a, N_{0}(2 r+t)\right)\right)
\end{gathered}
$$

for any $s>0, r \geq 0, a \in X$ and $t \geq 0$, where $N_{0}=a_{1}\left(1+2 a_{0}\right)$.

Then there exists a positive constant $c_{2}$ such that for any $\lambda>0$ and any nonnegative $\nu$-measurable function $f: X \rightarrow \mathbf{R}^{1}$ the following inequality holds:

$$
\begin{gathered}
\varphi(\lambda) \theta(\beta\{(x, t) \in X \times[0, \infty): \mathcal{K}(f d \nu)(x, t)>\lambda\}) \leq \\
\leq c_{2} \int_{X} \psi\left(\frac{f(x)}{\eta(\lambda)}\right) \sigma(x) d \nu(x) .
\end{gathered}
$$

Assume now that the nonnegative measurable kernel $k$ satisfies the following additional condition: there exist numbers $N \geq N_{0}, N_{0}=a_{1}\left(1+2 a_{0}\right)$ and $c^{\prime}$ such that

$$
k(a, y, t) \leq c^{\prime} k(x, y, \tau)
$$

when $y \in X \backslash B(a, r),(x, \tau) \in \hat{B}(a, N(r+t))$ for any $a \in X, r \geq 0, t \geq 0$.

Theorem 1.2. Let $\varphi \eta$ and $\psi$ be quasiconvex functions, let $\psi \in \Delta_{2}$, let the function $t^{-\alpha} \theta(t)$ decrease for some $\alpha \in(0,1)$, and let $k$ satisfy the condition (1.7).

Then the inequality (1.6) is equivalent to any of the following conditions: 
(i) there exist positive constants $\varepsilon$ and $c_{3}$ such that

$$
\begin{gathered}
\int_{X \backslash B(a, r)} \tilde{\psi}\left(\varepsilon \frac{\varphi(s) \eta(s)}{s} \frac{\theta(\beta \hat{B}(a, r+t))}{\sigma(y)} k(a, y, t)\right) \sigma(y) d \nu(y) \leq \\
\leq c_{3} \varphi(s) \theta(\beta \hat{B}(a, r+t))
\end{gathered}
$$

for arbitrary $s>0, r \geq 0, a \in X$ and $t \geq 0$;

(ii) there exist positive constants $\varepsilon$ and $c_{4}$ such that

$$
\begin{gathered}
\int_{X} \tilde{\psi}\left(\varepsilon \frac{\varphi(s) \eta(s)}{s} \frac{\theta(\beta \hat{B}(a, t))}{\sigma(y)} k(a, y, t)\right) \sigma(y) d \nu(y) \leq \\
\leq c_{4} \varphi(s) \theta(\beta \hat{B}(a, t))
\end{gathered}
$$

for any $s>0, a \in X$ and $t \geq 0$;

(iii) there exists a positive constant $c_{5}$ such that for any $a \in X, r \geq 0$, $t \geq 0$ and for any nonnegative $\nu$-measurable functions $F: X \rightarrow \mathbf{R}^{1}$, $\operatorname{supp} F \subset X \backslash B(a, r)$ we have

$$
\begin{array}{r}
\varphi(\mathcal{K}(F d \nu)(a, t)) \theta(\beta \hat{B}(a, r+t)) \leq \\
\leq c_{5} \int_{X} \psi\left(\frac{F(x)}{\eta(\mathcal{K}(F d \nu)(a, t)}\right) \sigma(y) d \nu(y) .
\end{array}
$$

Let $k: X \times X \rightarrow \mathbf{R}^{1}$ be a nonnegative measurable function satisfying the following condition: there exist numbers $N \geq N_{0}$ and $c^{\prime}>0$ such that $k(a, y) \leq c^{\prime} k(x, y)$ for any $a \in X, y \in X \backslash B(a, r)$, and $x \in B(a, N r)$.

For any positive function $\varrho: X \rightarrow \mathbf{R}^{1}$, locally summable with respect to measure $\nu$, it will be assumed below that

$$
\varrho E=\int_{E} \varrho(x) d \nu(x)
$$

for any $\nu$-measurable set $E \subset X$.

We have

Theorem 1.3. Let the functions $\varphi, \eta$, and $\psi$ satisfy the conditions of Theorem 1.2. Then the following statements are equivalent:

(i) there exists a positive constant $c_{6}$ such that for any $\lambda>0$ and for any measurable nonnegative function $f$

$$
\begin{gathered}
\varphi(\lambda) \theta\left(\varrho\left\{x \in X: \int_{X} k(x, y) f(y) d \nu(y)>\lambda\right\}\right) \leq \\
\leq c_{6} \int_{X} \psi\left(\frac{f(y)}{\eta(\lambda)}\right) \sigma(y) d \nu(y)
\end{gathered}
$$


for any $\lambda>0$ and for any measurable nonnegative function $f: X \rightarrow \mathbf{R}^{1}$;

(ii) there exist positive numbers $\varepsilon$ and $c_{7}$ such that

$$
\begin{gathered}
\int_{X \backslash B(a, r)} \tilde{\psi}\left(\varepsilon \frac{\varphi(s) \eta(s)}{s} \frac{\theta(\varrho B(a, r))}{\sigma(y)} k(a, y)\right) \sigma(y) d \nu(y) \leq \\
\leq c_{7} \varphi(s) \theta(\varrho B(a, r))
\end{gathered}
$$

for any $s>0, a \in X$, and $r \geq 0$.

The above-formulated results contain the solutions of problems of description of a set of weights ensuring in Orlicz spaces the validity of both weak and extra-weak weighted inequalities for transform (1.1) which are natural analogies of inequalities of the weak type $(p, q)$. Indeed, for $\varphi=\psi, \eta \equiv 1$ (1.6) becomes a weak type weighted inequality, while for $\varphi \equiv 1, \eta(\lambda)=\lambda$ we obtain an extra-weak type weighted inequality. It is understood that an inequality of the weak type $(\varphi, \varphi)$ is essentially stronger than an inequality of the extra-weak type $(\varphi, \varphi)$.

The solutions of similar problems in Lorentz spaces are derived in Section 3. Section 4 contains a discussion of the interesting corollaries of Theorems 1.2 and 1.3 for integral operators such as potentials and their generalizations, Poisson integrals and their generalizations, the Hardy operators, and others. Here we give a very brief survey only of the results preceding this paper.

The solution of a weak type two weight problem for Riesz potentials in Lebesgue spaces was obtained in [3], [4], the criterion found in [4] being more easily verifiable. The latter result was extended to the integrals on homogeneous type spaces in [5]. A similar problem was treated in [6] (see also [7], Theorems 6.1.1 and 6.1.2) in Lorentz spaces over $\mathbf{R}^{n}$ for integral transforms

$$
K f(x)=\int_{\mathbf{R}^{n}} k(x, y) f(y) d y .
$$

Subsequently in [8] generalizations were obtained for transforms of type (1.1) when $X=\mathbf{R}^{n}, d \nu(y)=d y$. More particular cases of generalized potentials and Poisson integrals were considered in [8] and [9], respectively. The latter deals with Lorentz spaces and the former with Lebesgue spaces. In Orlicz classes the problem of description of a set of weights ensuring the validity of weak type weighted inequalities was previously studied mainly for maximal functions [10], [11], [12] and the Hardy operator [13], [14].

\section{Proof of the Main Theorems}

In this section use will be made of some properties of quasiconvex functions satisfying the $\Delta_{2}$ condition, also of the covering lemma in homogeneous 
type spaces.

Lemma 2.1 ([11], p. 4). The following statements are equaivalent:

(i) $\varphi$ is a quasiconvex function;

(ii) there exists $c>1$ such that

$$
\frac{\varphi(s)}{s} \leq \frac{\varphi(c t)}{t} \text { for } s<t .
$$

Hence for quasiconvex functions $\varphi$ we immediately obtain the estimates

$$
\begin{aligned}
& \delta \varphi(t) \leq \varphi(c \delta t), \quad t \geq 0, \quad \delta>1, \\
& \varphi(\delta t) \leq \delta \varphi(c t), \quad t \geq 0, \quad \delta<1 .
\end{aligned}
$$

For convex functions the inequalities to be given above are valid when $c=1$.

Lemma 2.2. If $\omega$ is a Young function, then

$$
\omega\left(\frac{\tilde{\omega}(t)}{t}\right) \leq \tilde{\omega}(t), \quad t \geq 0 .
$$

Proof. By virtue of the equality $(\tilde{\omega})^{\sim}=\omega$ we have

$$
\omega\left(\frac{\tilde{\omega}(t)}{t}\right)=\sup _{s \geq 0} s\left(\frac{\tilde{\omega}(t)}{t}-\frac{\tilde{\omega}(s)}{s}\right) \leq \sup _{0 \leq s<t} s \frac{\tilde{\omega}(t)}{t} \leq \tilde{\omega}(t) .
$$

since the expression in the brackets is negative when $t<s$.

Lemma 2.3 ([11], p.17). Let $\psi$ satisfy the $\Delta_{2}$-condition. Then there exist $p>1$ and $c>1$ such that

$$
s^{-p} \psi(s) \leq c t^{-p} \psi(t)
$$

for $0 \leq t \leq s$.

Lemma 2.4. Let $E$ be a bounded set in $X$, and for each point $x \in E$ let a ball $B_{x}=B\left(x, r_{x}\right)$ be given such that $\sup _{x \in E} \operatorname{rad} B_{x}<\infty$. Then from the family $\left\{B_{x}\right\}_{x \in E}$ we can choose a (finite or infinite) sequence of pairwise disjoint balls $\left(B_{j}\right)_{j}$ for which $E \subset \cup_{j \geq 1} N_{0} B_{j}, N_{0}=a_{1}\left(1+2 a_{0}\right)$, and for each $B_{x} \in\left\{B_{x}\right\}_{x \in E}$ there exists a ball $B_{j_{0}}$ such that $x \in N_{0} B_{j_{0}}$ and $\operatorname{rad} B_{x} \leq 2 \operatorname{rad} B_{j_{0}}$.

Proof. Set $R_{1}=\sup _{x \in E} \operatorname{rad} B_{x}$. There obviously exists a ball $B_{1}=B_{x_{1}}$ from the family $\left\{B_{x}\right\}_{x \in E}$ provided that $\operatorname{rad} B_{1}>2^{-1} R_{1}$. If $x \notin N_{0} B_{1} \cap E$, then $B_{x} \cap B_{1}=\varnothing$. Indeed, making the opposite assumption that there exists a point $y \in B_{x} \cap B_{1}$, we will have

$$
\begin{aligned}
& d\left(x_{1}, x\right) \leq a_{1}\left(d\left(x_{1}, y\right)+d(y, x)\right)<a_{1}\left(\operatorname{rad} B_{x_{1}}+a_{0} d(x, y)\right)< \\
& <a_{1}\left(\operatorname{rad} B_{1}+a_{0} \operatorname{rad} B_{x}\right)<a_{1}\left(1+2 a_{0}\right) \operatorname{rad} B_{1}=N_{0} \operatorname{rad} B_{1},
\end{aligned}
$$


which leads to the contradiction.

Obviously, $\operatorname{rad} B_{x} \leq 2 \mathrm{rad} B_{1}$ for an arbitrary point $x \in N_{0} B_{1} \cap E$. Assuming now that $R_{2}=\sup _{x \in E \backslash N_{0} B_{1}} \operatorname{rad} B_{x}$, we can find a ball $B_{2}=B_{x_{2}}$

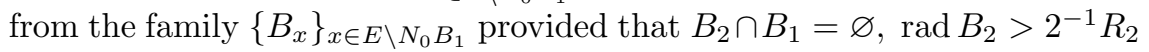
and $\operatorname{rad} B_{x} \leq 2 \operatorname{rad} B_{2}$ for each point $x \in\left(N_{0} B_{2} \cap E\right) \backslash N_{0} B_{1}$. Proceeding in this way, we arrive at the sequence $\left\{B_{j}\right\}_{j \geq 1}$ of nonintersecting balls. If this sequence is finite, then it will be the one we wanted to obtain.

Let the sequence be infinite. If we show that for each point $x \in E$ there exists a ball $B_{j}$ for which $x \in N_{0} B_{j}$, then setting $j_{0}$ equal to the minimal value among similar $j$ 's, we obtain the desired covering.

Assume the opposite. Let in $E$ there exists a point $x_{0} \in E$ such that $x_{0} \notin N_{0} B_{j}$ for every $j$. Then we will have $B_{x_{0}} \in\left\{B_{x}\right\}_{x \in E \backslash \cup_{j=1}^{n} N_{0} B_{j}}$ for any natural number $n$, and hence $\operatorname{rad} B_{x_{0}} \leq R_{n}<2 \operatorname{rad} B_{n}$ for each $n$.

On the other hand, it is obvious that $\cup_{x \in E} B_{x}$ is a bounded set, i.e. it is contained within some ball $B_{0}$. It therefore turns out that $\left(B_{j}\right)_{j \geq 1}$ is an infinite sequence of nonintersecting balls contained in $B_{0}$. Therefore $\operatorname{rad} B_{n} \rightarrow 0$ (see, for example, [17], p. 68). The latter result leads to the contradiction rad $B_{x_{0}}=0$.

Proof of Theorem 1.1. Fix the function $f \geq 0$ and $\lambda>0$. Without loss of generality it can be assumed that

$$
\frac{1}{c_{1} \theta(\beta(X \times[0, \infty))) \varphi(\lambda)} \int_{X} \psi\left(\frac{4 c_{1}}{\varepsilon} \frac{f(x)}{\eta(\lambda)}\right) \sigma(x) d \nu<1 .
$$

Otherwise we would have

$$
\begin{aligned}
\varphi(\lambda) \theta(\beta\{(x, t): & \mathcal{K}(f d \nu)(x, t)>\lambda\}) \leq \varphi(\lambda) \theta(\beta(X \times[0, \infty))) \leq \\
& \leq \frac{1}{c_{1}} \int_{X} \psi\left(\frac{4 c_{1}}{\varepsilon} \frac{f(x)}{\eta(\lambda)}\right) \sigma(x) d \nu
\end{aligned}
$$

and, since $\psi \in \Delta_{2}$, the proof is complete.

Assume $(x, t) \in E_{\lambda}$, where $E_{\lambda}=\left\{\left(x^{\prime}, t^{\prime}\right) \in X \times[0, \infty): \mathcal{K}(f d \nu)\left(x^{\prime}, t^{\prime}\right)\right.$ $>\lambda\}$. By virtue of $(2.6)$ for $(x, t)$ there exists a finite $r \geq 0$ such that

$$
\frac{1}{c_{1} \theta\left(\beta \widehat{B}\left(x, N_{0}(2 r+t)\right)\right) \varphi(\lambda)} \int_{X} \psi\left(\frac{4 c_{1}}{\varepsilon} \frac{f(x)}{\eta(\lambda)}\right) \sigma(x) d \nu<1 .
$$

If the greatest lower bound of $r$ is positive, then there exists a positive number $r_{0}=r_{0}(x, t)$ such that the inequalities

$$
\begin{aligned}
& \frac{1}{c_{1} \theta\left(\beta \widehat{B}\left(x, N_{0}\left(r_{0}+t\right)\right)\right) \varphi(\lambda)} \int_{X} \psi\left(\frac{4 c_{1}}{\varepsilon} \frac{f(x)}{\eta(\lambda)}\right) \sigma(x) d \nu \geq 1, \\
& \frac{1}{c_{1} \theta\left(\beta \widehat{B}\left(x, N_{0}\left(2 r_{0}+t\right)\right)\right) \varphi(\lambda)} \int_{X} \psi\left(\frac{4 c_{1}}{\varepsilon} \frac{f(x)}{\eta(\lambda)}\right) \sigma(x) d \nu<1
\end{aligned}
$$


are simultaneously fulfilled.

For such $r_{0}$ we would have by virtue of inequality (1.4) and condition (1.5)

$$
\begin{gathered}
\int_{X \backslash B\left(x, r_{0}\right)} f(y) k(x, y, t) d \nu=\frac{\lambda}{4 c_{1} \varphi(\lambda) \theta\left(\beta \widehat{B}\left(x, N_{0}\left(2 r_{0}+t\right)\right)\right)} \times \\
\times \int_{X \backslash B\left(x, r_{0}\right)} \frac{4 c_{1}}{\varepsilon} \frac{f(y)}{\eta(\lambda)} \varepsilon \frac{\varphi(\lambda) \eta(\lambda)}{\lambda} \frac{\theta\left(\beta \widehat{B}\left(x, N_{0}\left(2 r_{0}+t\right)\right)\right)}{\sigma(y)} k(x, y, t) \sigma(y) d \nu \leq \\
\leq \frac{\lambda}{4 c_{1} \varphi(\lambda) \theta\left(\beta \widehat{B}\left(x, N_{0}\left(2 r_{0}+t\right)\right)\right)} \int_{X \backslash B\left(x, r_{0}\right)} \psi\left(\frac{4 c_{1} f(x)}{\varepsilon \eta(\lambda)}\right) \sigma(x) d \nu+ \\
+\frac{\lambda}{4 c_{1} \varphi(\lambda) \theta\left(\beta \widehat{B}\left(x, N_{0}\left(2 r_{0}+t\right)\right)\right)} \times \\
\times \int_{X \backslash B\left(x, r_{0}\right)} \widetilde{\psi}\left(\varepsilon \frac{\varphi(\lambda) \eta(\lambda)}{\lambda} \frac{\theta\left(\beta \widehat{B}\left(x, N_{0}\left(2 r_{0}+t\right)\right)\right)}{\sigma(y)} k(x, y, t)\right) \sigma(y) d \nu \leq \\
\leq \frac{\lambda}{4}+\frac{\lambda}{4}=\frac{\lambda}{2} .
\end{gathered}
$$

But since $(x, t) \in E_{\lambda}$, the latter estimate implies

$$
\int_{B\left(x, r_{0}\right)} f(y) k(x, y, t) d \nu>\frac{\lambda}{2} .
$$

When the measure $\nu$ is concentrated at the point $x$, the above-mentioned greatest lower bound may turn out to be equal to zero. Then instead of (2.9) we have

$$
k(x, x, t) f(x) \nu\{x\}>\frac{\lambda}{2} .
$$

Therefore due to (1.4) and (1.5)

$$
\begin{gathered}
\left.\varphi(\lambda) \theta\left(\beta \widehat{B}\left(x, N_{0} t\right)\right)\right) \leq \frac{1}{2 c_{1}} \psi\left(\frac{4 c_{1}}{\varepsilon} \frac{f(x)}{\eta(\lambda)}\right) \sigma(x) \nu\{x\}+ \\
+\frac{1}{2 c_{1}} \widetilde{\psi}\left(\varepsilon \frac{\varphi(\lambda) \eta(\lambda)}{\lambda} \frac{\left.\theta\left(\beta \widehat{B}\left(x, N_{0} t\right)\right)\right)}{\sigma(x)} k(x, x, t)\right) \sigma(x) \nu\{x\} \leq \\
\leq \frac{1}{2 c_{1}} \int_{B(x, t)} \psi\left(\frac{4 c_{1}}{\varepsilon} \frac{f(x)}{\eta(\lambda)}\right) \sigma(y) d \nu(y)+\frac{1}{2} \varphi(\lambda) \theta\left(\beta \widehat{B}\left(x, N_{0} t\right)\right) .
\end{gathered}
$$


Hence

$$
\varphi(\lambda) \theta\left(\beta \widehat{B}\left(x, N_{0} t\right)\right) \leq \frac{1}{4 c_{1}} \int_{B(x, t)} \psi\left(\frac{4 c_{1}}{\varepsilon} \frac{f(y)}{\eta(\lambda)}\right) \sigma(y) d \nu(y) .
$$

Let us now consider the case when $\nu$ is not concentrated at the point $x$. Let $n$ be the greatest nonnegative integer for which

$$
\left.b \stackrel{\text { def }}{=} \lim _{r \rightarrow 0} \beta \hat{B}(r+t)\right)<2^{-n} \beta \widehat{B}\left(x, N_{0}\left(r_{0}+t\right)\right) ;
$$

$n$ may be equal to $\infty$ if $b=0$. For each $k, 0<k \leq n$, we set $r_{k}=\sup \{r$ : $\left.\beta \widehat{B}\left(x, N_{0}(r+t)\right) \leq 2^{-k} \beta \widehat{B}\left(x, N_{0}\left(r_{0}+t\right)\right)\right\}$. Then $\left(r_{k}\right)_{k}$ is a decreasing (finite or infinite) sequence and

$$
\begin{aligned}
\beta \widehat{B}\left(x, N_{0}\left(r_{k}+t\right)\right) & \leq 2^{-k} \beta \widehat{B}\left(x, N_{0}\left(r_{0}+t\right)\right) \leq \\
& \leq \beta \widehat{B}\left(x, N_{0}\left(2 r_{k}+t\right)\right) .
\end{aligned}
$$

Let $B_{k}=B\left(x, r_{k}\right), 0 \leq k \leq n$, and $B_{n+1}=\{x\}$. Since by the condition of the theorem $u^{-\alpha} \theta(u)$ decreases, we have

$$
\begin{gathered}
a_{k}^{p}=\frac{\beta \widehat{B}\left(x, N_{0}\left(r_{k}+t\right)\right)}{\theta\left(\beta \widehat{B}\left(x, N_{0}\left(r_{k}+t\right)\right)\right)} \cdot \frac{\theta\left(\beta \widehat{B}\left(x, N_{0}\left(r_{0}+t\right)\right)\right)}{\beta \widehat{B}\left(x, N_{0}\left(r_{0}+t\right)\right)}= \\
=\frac{\beta \widehat{B}\left(x, N_{0}\left(r_{k}+t\right)\right)}{\theta\left(\beta \widehat{B}\left(x, N_{0}\left(r_{k}+t\right)\right)\right)} \frac{\theta\left(\beta \widehat{B}\left(x, N_{0}\left(r_{0}+t\right)\right)\right)}{\left(\beta \widehat{B}\left(x, N_{0}\left(r_{0}+t\right)\right)\right)^{1-\alpha}\left(\beta \widehat{B}\left(x, N_{0}\left(r_{0}+t\right)\right)\right)^{\alpha}} \leq \\
\leq c\left(\frac{\beta \widehat{B}\left(x, N_{0}\left(r_{k}+t\right)\right)}{\beta \widehat{B}\left(x, N_{0}\left(r_{0}+t\right)\right)}\right)^{1-\alpha} \leq c\left(\frac{1}{2^{k}}\right)^{1-\alpha} .
\end{gathered}
$$

Because of this

$$
\sum_{k=0}^{n} a_{k} \leq c^{\frac{1}{p}} \sum_{k=0}^{\infty}\left(\frac{1}{2^{k}}\right)^{\frac{1-\alpha}{p}}=a<\infty
$$

Inequality (2.9) can now be rewritten as

$$
\frac{\lambda}{2} \sum_{k=0}^{n} \frac{a_{k}}{a} \leq \sum_{k=0}^{n} \int_{B_{k} \backslash B_{k+1}} k(x, y, t) f(y) d \nu,
$$

whence it follows that there exists $k_{0}, 0 \leq k_{0} \leq n$, such that

$$
\frac{\lambda}{2} \frac{a_{k_{0}}}{a}<\int_{B_{k_{0}} \backslash B_{k_{0}+1}} k(x, y, t) f(y) d \nu .
$$

Therefore

$$
\varphi(\lambda) \theta\left(\beta \widehat{B}\left(x, N_{0}\left(2 r_{k_{0}+1}+t\right)\right)\right) \leq
$$




$$
\begin{gathered}
\leq \frac{1}{2 c_{1}} \int_{B_{k_{0}} \backslash B_{k_{0}+1}} \frac{4 a c_{1}}{\varepsilon a_{k_{0}}} \frac{f(y)}{\eta(\lambda)} \varepsilon \frac{\varphi(\lambda) \eta(\lambda)}{\lambda} \frac{\theta\left(\beta \widehat{B}\left(x, N_{0}\left(2 r_{k_{0}+1}+t\right)\right)\right)}{\sigma(y)} \times \\
\quad \times k(x, y, t) \sigma(y) d \nu(y) \leq \frac{1}{2 c_{1}} \int_{B_{k_{0}}} \psi\left(\frac{4 a c_{1}}{\varepsilon a_{k_{0}}} \frac{f(y)}{\eta(\lambda)}\right) \sigma(y) d \nu+ \\
+\frac{1}{2 c_{1}} \int_{B_{k_{0}} \backslash B_{k_{0}+1}} \widetilde{\psi}\left(\varepsilon \frac{\varphi(\lambda) \eta(\lambda)}{\lambda} \frac{\theta\left(\beta \widehat{B}\left(x, N_{0}\left(2 r_{k_{0}+1}+t\right)\right)\right)}{\sigma(y)} \times\right. \\
\quad \times k(x, y, t)) \sigma(y) d \nu \leq \frac{1}{2 c_{1}} \int_{B_{k_{0}}} \psi\left(\frac{4 a c_{1}}{\varepsilon a_{k_{0}}} \frac{f(y)}{\eta(\lambda)}\right) \sigma(y) d \nu+ \\
+\frac{1}{2} \varphi(\lambda) \theta\left(\beta \widehat{B}\left(x, N_{0}\left(2 r_{k_{0}+1}+t\right)\right)\right)
\end{gathered}
$$

and as a result we have

$$
\varphi(\lambda) \theta\left(\beta \widehat{B}\left(x, N_{0}\left(2 r_{k_{0}+1}+t\right)\right)\right) \leq c a_{k_{0}}^{-p} \int_{B\left(x, r_{k_{0}}\right)} \psi\left(\frac{4 c_{1}}{\varepsilon} \frac{f(y)}{\eta(\lambda)}\right) \sigma(y) d \nu .
$$

Next, taking into account (2.12), we obtain the estimate

$$
\varphi(\lambda) \theta\left(\beta \widehat{B}\left(x, N\left(r_{k_{0}}+t\right)\right) \leq c a_{k_{0}}^{-p} \int_{B\left(x, r_{k_{0}}+t\right)} \psi\left(\frac{4 c_{1} f(y)}{\varepsilon \eta(\lambda)}\right) \sigma(y) d \nu\right.
$$

which by the definition of the number $a_{k_{0}}$ takes the form

$$
\begin{gathered}
\varphi(\lambda) \beta \widehat{B}\left(x, N_{0}\left(r_{k_{0}}+t\right)\right) \leq \\
\leq c \frac{\beta \widehat{B}\left(x, N_{0}\left(r_{0}+t\right)\right)}{\theta\left(\beta \widehat{B}\left(x, N_{0}\left(r_{0}+t\right)\right)\right)} \int_{B\left(x, r_{k_{0}}+t\right)} \psi\left(\frac{4 c_{1} f(y)}{\varepsilon \eta(\lambda)}\right) \sigma(y) d \nu .
\end{gathered}
$$

Rewrite (2.7) now as

$$
\theta\left(\beta \widehat{B}\left(x, N_{0}\left(r_{0}+t\right)\right)\right) \leq \frac{1}{c_{1} \varphi(\lambda)} \int_{X} \psi\left(\frac{4 c_{1}}{\varepsilon} \frac{f(y)}{\eta(\lambda)}\right) \sigma(y) d \nu .
$$

If $\theta^{-1}$ is defined by $\theta^{-1}(u)=\sup \{\tau: \theta(\tau) \leq u\}$, then $\theta\left(\theta^{-1}(u)\right) \leq u$ and $\theta\left(2 \theta^{-1}(u)\right) \geq u$, i.e., $\frac{u}{2} \leq \theta\left(\theta^{-1}(u)\right) \leq u$. Moreover, $\theta^{-1}(\theta(u))=\sup \{\tau$ : $\theta(\tau) \leq \theta(u)\} \geq u$. Therefore (2.15) will yield the estimate

$$
\begin{gathered}
\beta \widehat{B}\left(x, N_{0}\left(r_{0}+t\right)\right) \leq \theta^{-1}\left(\theta\left(\beta \widehat{B}\left(x, N_{0}\left(r_{0}+t\right)\right)\right)\right) \leq \\
\leq \theta^{-1}\left(\frac{1}{c_{1} \varphi(\lambda)} \int_{X} \psi\left(\frac{4 c_{1}}{\varepsilon} \frac{f(x)}{\eta(\lambda)}\right) \sigma(x) d \nu\right) .
\end{gathered}
$$


Thus

$$
\begin{gathered}
\theta\left(\theta^{-1}\left(\frac{1}{c_{1} \varphi(\lambda)} \int_{X} \psi\left(\frac{4 c_{1} f(x)}{\varepsilon \eta(\lambda)}\right) \sigma(x) d \nu\right)\right) \leq \\
\leq \frac{1}{c_{1} \varphi(\lambda)} \int_{X} \psi\left(\frac{4 c_{1} f(x)}{\varepsilon \eta(\lambda)}\right) \sigma(x) d \nu
\end{gathered}
$$

which by virtue of the fact that $\frac{\theta(u)}{u}$ decreases yields the estimate

$$
\begin{gathered}
\frac{\beta \widehat{B}\left(x, N_{0}\left(r_{0}+t\right)\right)}{\theta\left(\beta \widehat{B}\left(x, N_{0}\left(r_{0}+t\right)\right)\right)} \leq \\
\leq c \frac{\theta^{-1}\left(\frac{1}{c_{1} \varphi(\lambda)} \int_{X} \psi\left(\frac{4 c_{1}}{\varepsilon} \frac{f(y)}{\eta(\lambda)}\right) \sigma(y) d \nu\right)}{\theta\left(\theta^{-1}\left(\frac{1}{c_{1} \varphi(\lambda)} \int_{X} \psi\left(\frac{4 c_{1}}{\varepsilon} \frac{f(y)}{\eta(\lambda)}\right) \sigma(y) d \nu\right)\right)} \leq \\
\leq 2 c \frac{\theta^{-1}\left(\frac{1}{c_{1} \varphi(\lambda)} \int_{X} \psi\left(\frac{4 c_{1}}{\varepsilon} \frac{f(y)}{\eta(\lambda)}\right) \sigma(y) d \nu\right)}{\frac{1}{c_{1} \varphi(\lambda)} \int_{X} \psi\left(\frac{4 c_{1}}{\varepsilon} \frac{f(y)}{\eta(\lambda)}\right) \sigma(y) d \nu} \stackrel{\text { def }}{=} l(f, \lambda) .
\end{gathered}
$$

After the above manipulations condition (2.14) can be formulated as follows: for each $(x, t) \in E_{\lambda}$ there exists a ball $B_{x, t}$ such that $x$ is its center, $t \leq \operatorname{rad} B_{x, t}$, and

$$
\varphi(\lambda) \beta\left(N_{0} \widehat{B}_{x, t}\right) \leq c l(f, \lambda) \int_{B_{x, t}} \psi\left(\frac{4 c_{1}}{\varepsilon} \frac{f(y)}{\eta(\lambda)}\right) \sigma(y) d \nu .
$$

Now fix a ball $B_{0}$ and consider the sets $\widehat{B}_{0} \cap E_{\lambda}$ and $B_{0} \cap\{x: \mathcal{K}(f d \nu)(x, 0)$ $>\lambda\} \equiv B_{0} \cap E_{\lambda}^{0}$.

It is obvious that the latter set is contained in the former. For each $x \in E_{\lambda}^{0}$ we set $d(x)=\sup \left\{t:(x, t) \in \widehat{B}_{0} \cap E_{\lambda}\right\}$. It is easy to verify that $d(x)<2 \operatorname{rad} B_{0}$. For each $x \in B_{0} \cap E_{\lambda}^{0}$ there exist $t_{x} \geq \frac{2 d(x)}{N_{0}}$ such that $\left(x, t_{x}\right) \in \hat{B}_{0} \cap E_{\lambda}\left(N_{0}>2\right)$; consequently, for $\left(x, t_{x}\right)$, (2.16) is valid.

As a result we have the following situation: for each $x \in B_{0} \cap E_{\lambda}^{0}$ there exists a ball $B_{x}$ with center at the point $x$ such that $\operatorname{rad} B_{x}>\frac{d(x)}{N_{0}}$ and (2.16) is fulfilled for $B_{x}=B_{x, t}$.

If $\sup _{\operatorname{rad}} B_{x}=\infty$, then, clearly, there exists a ball $B_{1} \in\left\{B_{x}\right\}_{x \in E_{\lambda}^{0} \cap B_{0}}$ $x \in B_{0} \cap E_{\lambda}^{0}$

such that $E_{\lambda} \cap \widehat{B}_{0} \subset \widehat{B}_{1}$.

If sup rad $B_{x}<\infty$, then, due to Lemma 2.4 , from the family $\left\{B_{x}\right\}_{x \in E_{\lambda}^{0}} \cap$ $B_{0}$ covering the bounded set $E_{\lambda}^{0} \cap B_{0}$ we can choose a sequence $\left(B_{j}\right)$ of nonintersecting balls for which $\cup_{j \geq 1} N_{0} B_{j} \supset E_{\lambda}^{0} \cap B_{0}$ and (2.16) holds.

It will be shown that $\left(\widehat{N_{0} B_{j}}\right)_{j \geq 1}$ covers the set $E_{\lambda} \cap B_{0}$. To this end we prove that each $(x, d(x)) \in \cup_{j \geq 1} \widehat{N_{0} B_{j}}$. Indeed, if $x$ is the center of some 
ball $B_{j}$, then there is nothing to prove. Let $x$ not be the center of $B_{j}$; then by Lemma 2.4 for $B_{x}$ there exists a ball $B_{j}$ such that $x \in N_{0} B_{j}$ and $\operatorname{rad} B_{x} \leq 2 \operatorname{rad} B_{j}$. Therefore $d(x)<N_{0} \operatorname{rad} B_{x} \leq 2 N_{0} \operatorname{rad} B_{j}$ or, which is the same, $(x, d(x)) \in \widehat{N_{0} B_{j}}$.

On account of the foregoing reasoning we can derive estimates

$$
\begin{gathered}
\varphi(\lambda) \beta\left\{(x, t) \in \widehat{B_{0}}: \mathcal{K}(f d \nu)(x, t)>\lambda\right\} \leq \sum_{j=1}^{\infty} \varphi(\lambda) \beta \widehat{N_{0} B_{j}} \leq \\
\leq c l(f, \lambda) \sum_{j=1}^{\infty} \int_{B_{j}} \psi\left(\frac{4 c_{1}}{\varepsilon} \frac{f(y)}{\eta(\lambda)}\right) \sigma(y) d \nu \leq \\
\leq c l(f, \lambda) \int_{X} \psi\left(\frac{4 c_{1}}{\varepsilon} \frac{f(y)}{\eta(\lambda)}\right) \sigma(y) d \nu \leq \\
\leq c \varphi(\lambda) \theta^{-1}\left(\frac{1}{c_{1} \varphi(\lambda)} \int_{X} \psi\left(\frac{4 c_{1}}{\varepsilon} \frac{f(y)}{\eta(\lambda)}\right) \sigma(y) d \nu\right)
\end{gathered}
$$

which yield

$$
\begin{gathered}
\theta\left(\beta\left\{(x, t) \in \widehat{B_{0}}: \mathcal{K}(f d \nu)(x, t)>\lambda\right\}\right) \leq \\
\leq \theta\left(c \theta^{-1}\left(\frac{1}{c_{1} \varphi(\lambda)} \int_{X} \psi\left(\frac{4 c_{1}}{\varepsilon} \frac{f(x)}{\eta(\lambda)}\right) \sigma(x) d \nu\right)\right) .
\end{gathered}
$$

Taking into account $\psi \in \Delta_{2}$ and $u^{-\alpha} \psi(u) \downarrow$, from the latter estimate we obtain the inequality

$$
\begin{gathered}
\theta\left(\beta\left\{(x, t) \in \widehat{B_{0}}: \mathcal{K}(f d \nu)(x, t)>\lambda\right\}\right) \leq \\
\leq c^{\alpha} \theta\left(\theta^{-1}\left(\frac{1}{c_{1} \varphi(\lambda)} \int_{X} \psi\left(\frac{4 c_{1}}{\varepsilon} \frac{f(x)}{\eta(\lambda)}\right) \sigma(x) d \nu\right)\right) \leq \\
\leq \frac{c}{\varphi(\lambda)} \int_{X} \psi\left(\frac{f(x)}{\eta(\lambda)}\right) \sigma(x) d \nu .
\end{gathered}
$$

If we now assume that $\operatorname{rad} B_{0}$ tends to infinity, we obtain (1.6).

Consider the case $d \beta=\varrho d \nu \otimes \delta_{0}$, where $\delta_{0}$ is the Dirac measure supported at the origin and

$$
k(x, y, t)= \begin{cases}k(x, y), & t=0 \\ 0, & t>0 .\end{cases}
$$

In that case due to Theorem 1.1 we have

Corollary 2.1. Let the functions $\varphi, \eta$ and $\psi$ satisfy the conditions of Theorem 1.1. It is further assumed that there exist positive $\varepsilon$ and $c_{1}$ such 
that

$$
\begin{gathered}
\int_{X \backslash B(a, r)} \widetilde{\psi}\left(\varepsilon \frac{\varphi(s) \eta(s)}{s} \frac{\theta\left(\varrho B\left(a, 2 N_{0} r\right)\right)}{\sigma(y)} k(a, y)\right) \sigma(y) d \nu \leq \\
c_{1} \varphi(s) \theta\left(\varrho B\left(a, 2 N_{0} r\right)\right)
\end{gathered}
$$

for any $s>0, r \geq 0$ and $a \in X$.

In that case there exists $c_{2}>0$ such that the inequality

$$
\begin{aligned}
\varphi(\lambda) \theta(\varrho\{x & \left.\left.\in X: \int_{X} k(x, y) f(y) d \nu(y)>\lambda\right\}\right) \leq \\
& \leq c_{2} \int_{X} \psi\left(\frac{f(x)}{\eta(\lambda)}\right) \sigma(x) d \nu
\end{aligned}
$$

holds for any $\lambda>0$ and any nonnegative measurable function $f: X \rightarrow \mathbf{R}^{1}$.

It is time to make some remarks. Taking a closer look at the proof of Theorem 1.1, we readily find that if $\beta \widehat{B}(x, r)$ is continuous with respect to $r$ for each $x \in X$, the factor 2 in condition (1.5) can be omitted.

Moreover, if the space $(X, d, \mu)$ possesses the Besicovitch property (consisting in the fact that for every bounded set $E$ any family $\{B(y, r(y))\}_{y \in E}$ of balls contains a countable (or finite) subfamily $\left\{B_{n}\right\}=\left\{B\left(y_{n}, r\left(y_{n}\right)\right\}\right.$, $n \in N$, such that $E \subset \cup B_{n}$ and $\sum \chi_{B_{n}} \leq c$, where $\chi_{B_{n}}$ is the characteristic function of the set $B_{n}$ ), then in Corollary 2.1 we can set $N_{0}=1$.

Finally we remark that for $\varphi(\lambda)=\psi(\lambda)=\lambda^{p}, \eta \equiv 1, \theta(u)=u^{\frac{p}{q}}, X=\mathbf{R}^{n}$ Corollary 2.1 becomes the particular case of Theorem 6.1.1 from [7], p. 171.

The proof of Theorem 1.2 rests on a number of lemmas.

Lemma 2.5. Let $\theta$ be any increasing function, and let the kernel $k$ satisfy condition (1.7). If condition (1.6) is fulfilled, then there exists a constant $c>0$ such that for any $a \in X, r \geq 0, t \geq 0$, and any nonnegative measurable function $F: X \rightarrow \mathbf{R}^{1}$, supp $F \subset X \backslash B(a, r)$ we have the inequality

$$
\begin{gathered}
\varphi(\mathcal{K}(F d \nu)(a, t)) \theta(\beta \widehat{B}(a, N(r+t))) \leq \\
\leq c \int_{X} \psi\left(\frac{c^{\prime} F(x)}{\eta(\mathcal{K}(F d \nu)(a, t))}\right) \sigma(x) d \nu,
\end{gathered}
$$

where $N$ and $c^{\prime}$ are the constants from condition (1.7).

Proof. Fix $a \in X, r \geq 0, t \geq 0$, and $F: X \rightarrow \mathbf{R}^{1}$ assuming that $\operatorname{supp} F \subset$ $X \backslash B(a, r)$. Inequality (2.17) is obtained if in (1.6) we set $f=c^{\prime} F$ and $\lambda=\mathcal{K}(F d \nu)(a, t)$.

It is sufficient only to note that the inclusion

$$
\widehat{B}(a, N(r+t)) \subset\left\{(x, \tau): \mathcal{K}\left(c^{\prime} F d \nu\right)(x, \tau) \geq \mathcal{K}(F d \nu)(a, t)\right\}
$$


holds by virtue of condition (1.7).

Lemma 2.6. Let $\varphi \eta$ and $\psi$ be quasiconvex functions, and let $k$ be a nonnegative kernel. Then condition (2.17) with the constants $c, c^{\prime}$, and $N$ implies the existence of $\varepsilon$ and $c_{1}$ such that the inequality

$$
\begin{gathered}
\int_{X \backslash B(a, r)} \widetilde{\psi}\left(\varepsilon \frac{\varphi(\lambda) \eta(\lambda)}{\lambda} \frac{\theta(\beta \widehat{B}(a, N(r+t)))}{\sigma(y)} k(a, y, t)\right) \sigma(y) d \nu(y) \leq \\
\leq c_{1} \varphi(\lambda) \theta(\beta \widehat{B}(a, N(r+t)))
\end{gathered}
$$

holds for any $\lambda>0, r \geq 0, t \geq 0$, and $a \in X$.

Proof. It is obvious that (2.17) is fulfilled for $\sigma_{1}=\sigma+\delta$, too, if $\delta>0$. Let $a \in X, r \geq 0, t \geq 0, \lambda>0$ be the fixed constants. Due to (1.8) it can be assumed without loss of generality that the function $\psi$ is convex. For $M>0$ we define the value

$$
I=\int_{D} \widetilde{\psi}\left(\varepsilon \frac{\varphi(\lambda) \eta(\lambda)}{\lambda} \frac{\theta(\beta \widehat{B}(a, N(r+t))}{\sigma_{1}(y)} k(a, y, t)\right) \sigma_{1}(y) d \nu,
$$

where $D=B(a, R) \backslash B(a, r) \cap\{y \in X: k(a, y, t)<M\}, R>r$, while the constant $\varepsilon$ will be appropriately chosen later.

We introduce the notation

$$
\begin{gathered}
T_{\delta}(y)=\varepsilon \frac{\varphi(\lambda) \eta(\lambda)}{\lambda} \frac{\theta(\beta \widehat{B}(a, N(r+t)))}{\sigma_{1}(y)} k(a, y, t), \\
g(y)=\frac{\widetilde{\psi}\left(T_{\sigma}(y)\right)}{T_{\sigma}(y)} \varepsilon \eta(\lambda) \chi_{D}(y),
\end{gathered}
$$

allowing us to write

$$
I=\varphi(\lambda) \theta(\beta \widehat{B}(a, N(r+t))) \frac{\mathcal{K}(g d \nu)(a, t)}{\lambda} .
$$

Our next step is to show that for sufficiently small $\varepsilon$ 's the value $I$ is finite. If $\lim _{t \rightarrow \infty} \frac{\psi(t)}{t}=\infty$, then $\widetilde{\psi}$ is finite everywhere and thus

$$
I \leq \widetilde{\psi}\left(\varepsilon \frac{\varphi(\lambda) \eta(\lambda)}{\lambda} \frac{\theta(\beta \widehat{B}(a, N(r+t)))}{\delta} M\right) \int_{B(a, R)} \sigma_{1}(x) d \nu<\infty
$$

for any $\varepsilon>0$.

Let now $\psi(t) \leq A t, A>0$. Then from the condition (2.17) we obtain

$$
\begin{aligned}
& \varphi(\mathcal{K}(F d \nu)(a, t)) \eta(\mathcal{K}(F d \nu)(a, t)) \theta(\beta \widehat{B}(a, N(r+t))) \leq \\
& \leq c \int_{X} F(x) \sigma(x) d \nu .
\end{aligned}
$$


Set

$$
l=\left\|\chi_{X \backslash B(a, r)} \frac{k(a, \cdot, t)}{\sigma_{1}}\right\|_{L^{\infty}} .
$$

From the definition of the norm in $L^{\infty}$ it follows that there exists a measurable set $E \subset X \backslash B(a, r), \nu E>0$ such that

$$
\frac{k(a, y, t)}{\sigma_{1}(y)}>\frac{l}{2}
$$

for $y \in E$.

Set in $(2.20)$

$$
F(y)=\frac{\lambda}{\nu E k(a, y, t)} \chi_{E}(y) .
$$

Recall that $a$ and $t$ are fixed. Obviously, $\mathcal{K}(F d \nu)(a, t)=\lambda$ and hence by virtue of (2.20) we obtain the estimate

$$
\frac{\varphi(\lambda) \eta(\lambda)}{\lambda} \theta(\beta \widehat{B}(a, N(r+t))) \leq \frac{c}{\nu E} \int_{E} \frac{\sigma(y)}{k(a, y, t)} d \nu \leq \frac{2 c}{l},
$$

which yields

$$
\frac{\varphi(\lambda) \eta(\lambda)}{\lambda} \theta(\beta \widehat{B}(a, N(r+t))) \chi_{X \backslash B(a, r)} \frac{k(a, y, t)}{\sigma_{1}(y)}<c,
$$

where the constant does not depend on $\lambda, r, t$, and $a$.

Thus we conclude that

$$
I \leq \widetilde{\psi}(\varepsilon c) \int_{B(a, r)} \sigma_{1}(y) d \nu .
$$

If now $\varepsilon$ is so small that $\widetilde{\psi}(c \varepsilon)<\infty$, then the value $I$ will be finite for the respective $\varepsilon$.

Now it will be shown that

$$
I \leq b \varphi(\lambda) \theta(\beta \widehat{B}(a, N(r+t)))+c b \int_{X} \psi\left(\frac{c^{\prime} g(y)}{\eta(\lambda)}\right) \sigma_{1} d \nu,
$$

where the constants $b, c$ and $c^{\prime}$ do not depend on $\lambda, r$, and $t$.

Let $a \in X$ and $t \geq 0$ be such that $\mathcal{K}(g d \nu)(a, t)<b \lambda$, where the constant $b$ is such that

$$
\frac{\varphi(s) \eta(s)}{s} \leq b \frac{\varphi(u) \eta(u)}{u}
$$

for $b s<u$ (see Lemma 2.1).

Then evidently (2.19) will yield

$$
I \leq b \varphi(\lambda) \theta(\beta \widehat{B}(a, N(r+t))) .
$$

Let now $\mathcal{K}(g d \nu)(a, t)>b \lambda$. 
Using (2.22) and condition (2.17), from (2.19) we obtain the estimate

$$
\begin{gathered}
I=\varphi(\lambda) \theta(\beta \widehat{B}(a, N(r+t))) \frac{\mathcal{K}(g d \nu)(a, t)}{\lambda} \leq \\
\leq b \frac{\varphi(\mathcal{K}(g d \nu)(a, t)) \eta(\mathcal{K}(g d \nu)(a, t))}{\eta(\lambda)} \theta(\beta \widehat{B}(a, N(r+t))) \leq \\
\leq c b \frac{\eta(\mathcal{K}(g d \nu)(a, t))}{\eta(\lambda)} \int_{X} \psi\left(\frac{c^{\prime} g(x)}{\eta(\mathcal{K}(g d \nu)(a, t))}\right) \sigma_{1}(x) d \nu .
\end{gathered}
$$

Since the function $\psi$ is convex, estimating the right-hand part of the latter inequality by means of (2.2) we conclude that

$$
I \leq c b \int_{X} \psi\left(\frac{c^{\prime} g(x)}{\eta(\lambda)}\right) \sigma_{1}(x) d \nu
$$

Thus we have shown that inequality (2.21) is valid.

Rewrite (2.21) as

$$
\begin{aligned}
& I \leq b \varphi(\lambda) \theta(\beta \widehat{B}(a, N(r+t)))+ \\
& +c b \int_{X} \psi\left(c^{\prime} \varepsilon \frac{\widetilde{\psi}\left(T_{\delta}(y)\right)}{T_{\delta}(y)}\right) \sigma_{1}(y) d \nu .
\end{aligned}
$$

Let $\varepsilon$ be chosen so small that $c^{\prime} \varepsilon<1$. Then, by virtue of the assumption that the function $\psi$ is convex and taking into account (2.3) and (2.4), from (2.23) we have

$$
\begin{gathered}
\int_{D} \widetilde{\psi}\left(T_{\delta}(y)\right) \sigma(y) d \nu \leq b \varphi(\lambda) \theta(\beta \widehat{B}(a, N(r+t)))+ \\
+c c^{\prime} b \varepsilon \int_{D} \widetilde{\psi}\left(T_{\delta}(y)\right) \sigma_{1}(y) d \nu .
\end{gathered}
$$

If $\varepsilon$ is so small that $c c^{\prime} b \varepsilon<1$, then the latter inequality implies

$$
\int_{D} \widetilde{\psi}\left(T_{\delta}(y)\right) \sigma_{1}(y) d \nu \leq c \varphi(\lambda) \theta(\beta \widehat{B}(a, N(r+t))) .
$$

Passing here to the limit when $R \rightarrow \infty, M \rightarrow \infty$, and $\delta \rightarrow 0$, we obtain the desired inequality (2.18).

Lemma 2.7. Let the kernel $k$ satisfy condition (1.7), and let inequality (1.9) be fulfilled. Then (1.5) is valid.

Proof. Replace $t$ by $N_{0}(2 r+t)$ in condition (1.9) and take into consideration that by virtue of condition (1.7) we have

$$
k(a, y, t) \leq c^{\prime} k\left(a, y, N_{0}(2 r+t)\right)
$$

for any $y \in X \backslash B(a, r)$. 
Proof of Theorem 2.2. The proof is accomplished by the diagram

$$
\begin{array}{ccc}
(1.6) & \Rightarrow & (1.10) \\
\Uparrow & & \Downarrow \\
(1.9) & \Leftarrow & (1.8)
\end{array}
$$

By Lemma $2.5(1.6) \Rightarrow(1.10)$. Then by Lemma $2.6(1.10) \Rightarrow(1.8)$. When $r=0$, condition (1.8) yields (1.9). Next, by Lemma 2.7 we obtain (1.5). Finally, using Theorem 1.1, we ascertain that the implication (1.5) $\Rightarrow(1.6)$ is valid.

We make some remarks connected with the proof of Theorem 1.3. If $k(x, y, t)=k(x, y), d \beta=\varrho d \mu \otimes \delta_{0}$ Lemmas 2.5 and 2.6 can be reformulated in the respective manner. Further, proceeding from Corollary 2.1 and following the proof of Theorem 2.2, we ascertain that Theorem 1.3 is valid.

\section{Criteria of General Weak Type Weighted Inequalities in LORENTZ SpaCES}

Let $(Y, \nu)$ be a space with a positive $\sigma$-additive measure $\nu$. When $1 \leq$ $p \leq \infty, 1 \leq s \leq \infty$, the Lorentz space $L_{\nu}^{p s}$ is the space of all $\nu$-measurable functions $f$ for which $\|f\|_{L^{p s}(Y, \nu)}<\infty$, where

$$
\begin{aligned}
\|f\|_{L^{p s}(Y, \nu)}= & \left(s \int_{0}^{\infty}(\nu\{y \in Y:|f(y)|>\tau\})^{\frac{s}{p}} \tau^{s-1} d \tau\right)^{\frac{1}{s}} \\
& \text { if } 1 \leq p<\infty, \quad 1 \leq s<\infty
\end{aligned}
$$

and

$$
\|f\|_{L^{p s}(Y, \nu)}=\sup \tau(\{y \in Y:|f(y)|>\tau\})^{\frac{1}{p}} \quad \text { if } \quad 1 \leq p<\infty, \quad s=\infty .
$$

If $1<p<\infty$ and $1 \leq s \leq \infty$, or $p=s=1$, or $p=s=\infty$, then $L^{p s}(Y, \nu)$ is a Banach space with norm equivalent to $\|\cdot\|_{L^{p s}(Y, \nu)}$.

In the sequel $X$ will denote a homogeneous type space, $\beta$ a positive measure given on the product of $\sigma$-algebras generated by balls from $X$ and by intervals from $[0, \infty)$, and $\nu$ a finite positive measure on $X$.

Theorem 3.1. Let $1 \leq s \leq p<q<\infty$, and let $k: X \times X \times[0, \infty) \rightarrow \mathbf{R}^{1}$ be an arbitrarily chosen nonnegative kernel. In that case, if there exists a number $c_{1}>0$ such that the inequality

$$
\left(\beta \widehat{B}\left(a, N_{0}(2 r+t)\right)^{\frac{1}{q}}\left\|\chi_{X \backslash B(a, r)} \frac{k(a, \cdot, t)}{\sigma}\right\|_{L^{p^{\prime} s^{\prime}}(X, \sigma d \nu)} \leq c_{1}\right.
$$


holds for any $a \in X, \quad r \geq 0, t \geq 0$, then there exists a positive constant $c_{2}$ such that

$$
\begin{gathered}
\beta\{(x, t) \in X \times[0, \infty): \mathcal{K}(f d \nu)(x, t)>\lambda\} \leq \\
\leq c_{2} \lambda^{-q}\|f\|_{L^{p s}(X, \nu)}^{q}
\end{gathered}
$$

for any measurable nonnegative $f: X \rightarrow \mathbf{R}^{1}$ and $\lambda>0$.

Theorem 3.2. Let $1 \leq s \leq p<q<\infty$, and let the kernel $k$ satisfy condition (1.7). Then the folowing statements are equivalent:

(i) (3.2) is fulfilled;

(ii) there exists a positive constant $c$ such that

$$
\left(\beta \widehat{B}(a,(2 r+t))^{\frac{1}{q}}\left\|_{X \backslash B(a, r)} \frac{k(a, \cdot, t)}{\sigma}\right\|_{L^{p^{\prime} s^{\prime}}(X, \sigma d \nu)} \leq c\right.
$$

for any $a \in X, \quad r \geq 0, \quad t \geq 0$;

(iii) there exists a number $c_{1}>0$ such that

$$
\left(\beta \widehat{B}(a, t)^{\frac{1}{q}}\left\|\frac{k(a, \cdot, t)}{\sigma}\right\|_{L^{p^{\prime} s^{\prime}}(x, \sigma d \nu)} \leq c_{1} .\right.
$$

The proofs of these theorems are accomplished in the manner described in Section 2 using the technique from [7], Chapter 6, and we therefore leave

them out. Note that the solution of the two-weight problem in the sense of [3] was previously derived in [19] in Lebesgue space for a fractional integral over a homogenous type space.

\section{General Weak Type Inequalities for Classical Operators}

In this section we discuss some specific examples for which the results of the previous sections are valid.

Consider the kernel

$$
k(x, y, t)=(\mu B(x, d(x, y)+t))^{-\delta}, \quad \delta>0 .
$$

It is easy to verify that it satisfies condition (1.7). Let $y \in X \backslash B(a, r)$ and $(x, \tau) \in \widehat{B}(a, N(r+t))$, where $N$ is an arbitrary positive number. It is sufficient to show the inclusion

$$
B(x, d(x, y)+\tau) \subset B(a, c(d(a, y)+t)) .
$$


Indeed, assuming that $z \in B(x, d(x, y)+\tau)$, we obtain a chain of inequalities

$$
\begin{gathered}
d(a, z) \leq a_{1}(d(a, x)+d(x, z)) \leq a_{1}(N(r+t)+d(x, z)) \leq \\
\leq a_{1} N(r+t)+a_{1} d(x, y)+a_{1} \tau \leq 3 a_{1} N(r+t)+ \\
+a_{1}^{2}(d(x, a)+d(a, y)) \leq 3 a_{1} N(r+t)+a_{1}^{2} a_{0} d(a, x)+ \\
+a_{1}^{2} d(a, y) \leq\left(3 a_{1}+a_{1}^{2} a_{0}\right) N(r+t)+a_{1}^{2} d(a, y) \leq \\
\leq\left(3 a_{1}+a_{1}^{2} a_{0}\right) N t+\left(\left(3 a_{1}+a_{1}^{2} a_{0}\right) N+a_{1}^{2}\right) d(a, y) \leq \\
\leq\left(\left(3 a_{1}+a_{1}^{2} a_{0}\right) N+a_{1}^{2}\right)(d(a, y)+t) .
\end{gathered}
$$

Thus condition (1.7) is fulfilled. For such kernels we have Theorems 2.2 and 3.2 and hence we obtain the solution of the general weak type weight problem in Orlicz and Lorentz spaces for classical operators such as Riesz potentials, Poisson integrals, and others.

Let $X=R^{n}, d$ a Euclidean distance, $\mu$ a Lebesgue measure, and

$$
T_{\gamma} f(x, t)=\int_{R^{n}} \frac{f(y)}{(|x-y|+t)^{n-\gamma}} d \nu, \quad 0<\gamma<n .
$$

a generalized potential. Theorem 2.2 yields a solution of the general weaktype weight problem for $T_{\gamma}$ in Orlicz spaces. It was previously solved in Lorentz space in [6] (see also [7], Theorem 6.5.1).

Now consider the Poisson integral in the upper half-space

$$
\mathbb{P} f(x, y)=\int_{R^{n}} f(y) \mathcal{P}(x-y, t) d y,
$$

where $\mathcal{P}(x, t)=c_{n} t\left(t^{2}+|x|^{2}\right)^{-\frac{n+1}{2}}$ is the Poisson kernel for $R_{+}^{n+1}$. The criterion of a two-weight inequality of the weak type $(p, q)$ was established in [10]. From Theorem 2.2 we obtain

Corollary 4.1. Let $\varphi \eta$ and $\psi$ be quasiconvex functions, $\psi \in \Delta_{2}$, and let the function $t^{-\alpha} \theta(t)$ decrease for some $\alpha \in(0,1)$. Then the following statements are equivalent:

(i) there exists a constant $c_{1}>0$ such that

$$
\begin{gathered}
\varphi(\lambda) \theta\left(\beta\left\{(x, t) \in R^{n} \times[0, \infty): \frac{1}{t} \mathbb{P} f(x, t)>\lambda\right\}\right) \leq \\
c_{1} \int_{R^{n}} \psi\left(\frac{f(x)}{\eta(\lambda)}\right) \sigma(x) d \nu
\end{gathered}
$$

for any $\lambda>0$ and any nonnegative measurable function $f: \mathbf{R}^{n} \rightarrow \mathbf{R}^{1}$; 
(ii) there exist positive constants $\varepsilon$ and $c_{2}$ such that

$$
\begin{gathered}
\int_{R^{n} \backslash B(a, r)} \widetilde{\psi}\left(\varepsilon \frac{\varphi(\lambda) \eta(\lambda)}{\lambda} \frac{\theta(\beta \widehat{B}(a, r+t))}{\sigma(y)} \frac{1}{t} \mathcal{P}(a-y, t)\right) \sigma(y) d \nu \leq \\
\leq c_{2} \varphi(\lambda) \theta(\beta \widehat{B}(a, r+t))
\end{gathered}
$$

for any $\lambda>0, a \in R^{n}, r \geq 0, t \geq 0$;

(iii) there exists positive constants $\varepsilon$ and $c_{3}$ such that

$$
\begin{gathered}
\int_{R^{n}} \widetilde{\psi}\left(\varepsilon \frac{\varphi(\lambda) \eta(\lambda)}{\lambda} \frac{\theta(\beta \widehat{B}(a, t))}{\sigma(y)} \frac{1}{t} \mathcal{P}(a-y, t)\right) \sigma(y) d \nu \leq \\
\leq c_{3} \varphi(\lambda) \theta(\beta \widehat{B}(a, t))
\end{gathered}
$$

for any $\lambda>0, a \in R^{n}$ and $t \geq 0$.

Let now $X=[0, \infty), d$ a Euclidean distance, $\mu$ a Lebesgue measure, and

$$
k(x, y)=\left\{\begin{array}{lll}
1 & \text { for } \quad x>y \\
0 & \text { for } \quad x \leq y
\end{array}\right.
$$

Then for the Hardy transform $f \mapsto \int_{0}^{x} f(y) d \nu$ Theorem 2.3 yields the criterion of validity of the weak-type inequality figuring in this theorem. This criterion is written in the form

$$
\int_{0}^{x} \widetilde{\psi}\left(\varepsilon \frac{\varphi(\lambda) \eta(\lambda)}{\lambda} \frac{\theta(\varrho(x, \infty))}{\sigma(y)}\right) \sigma(y) d \nu \leq c \varphi(\lambda) \theta(\varrho(x, \infty)) .
$$

\section{REFERENCES}

1. J. O. Strömberg and A. Torchinski, Weighted Hardy spaces. Lecture Notes in Math. 1381, Springer-Verlag, New York, 1989.

2. J. O. Strömberg, Bounded mean oscillation with Orlicz norms and duality of Hardy spaces. Indiana Univ. Math. J. 28(1979), 511-544.

3. E. T. Sawyer, A two-weight weak-type inequality for fractional integrals. Trans. Amer. Math. Soc. 281(1984), 339-345.

4. M. Gabidzashvili, Weighted inequalities for anisotropic potentials. (Russian) Trudy Tbiliss. Mat. Inst. Razmadze Akad. Nauk Gruzin. SSR 89(1986), 25-36.

5. I. Genebashvili, Carleson measures and potentials defined on spaces of the homogeneous type. (Russian) Soobshch. Akad. Nauk Gruz. SSR 135(1989), No. 3, 505-508. 
6. V. Kokilashvili and M. Gabidzashvili, Two-weight weak-type inequalities for fractional-type integrals. Preprint, No. 45, Math. Inst. Czech. Acad. Sci., Prague, 1989.

7. V. Kokilashvili and M. Krbec, Weighted inequalities in Lorentz and Orlicz spaces. World Scientific, Singapore, New Jersey, London, Hong Kong, 1991.

8. V.M. Kokilashvili, On the weight problem for integrals with positive kernels. Soobshch. Akad. Nauk Gruz. SSR 140(1990), No.3, 469-471.

9. M. Gabidzashvili, I. Genebashvili, and V. Kokilashvili, Two-weight inequalities for generalized potentials. (Russian) Trudy Mat. Inst. Steklov. 192(1991).

10. E. Sawyer and R. Wheeden, Carleson conditions for the Poisson integral. Indiana Univ. Math. J. 40(1991), No. 2, 639-676.

11. A. Carbery, S.Y. Chang, and J. Garnett, Weights and L $\log L$. Pacific J. Math. 120(1985), No.1, 33-45.

12. R.J. Bagby, Weak bounds for the maximal function in weighted Orlicz spaces. Stud. Math. 95(1990), 195-204.

13. D. Gallardo, Weighted weak type integral inequalities for the HardyLittlewood maximal operator. Izrael J. Math. 67(1989), No.1, 95-108.

14. L. Pick, Two-weight weak type maximal inequalities in Orlicz classes. Stud. Math. 100(1991), No.3, 206-218.

15. A. Gogatishvili and L. Pick, Weak and extra-weak type inequalities for the maximal operator and Hilbert transform, to appear in Czech. Math. $J$.

16. A. Gogatishvili, General weak-type inequalities for maximal operators and singular integrals. Proc. A. Razmadze Math. Inst. Georgian Acad. Sci. 101(1992), 47-63.

17. R. R. Coifman and G. Weiss, Analyse harmonique noncommutative sur certains espaces homogenes. Lecture Notes in Math. 242, Springer Verlag, Berlin and New York, 1971.

18. Pan Wenjie, Fractional integrals on spaces of homogeneous type. Approx. Theory and Its Appl. 8(1992), No.1, 1-15.

(Received 21.08.1992)

A.Razmadze Mathematical Institute

Georgian Academy of Sciences

1 Z.Rukhadze St., Tbilisi 380093

Republic of Georgia 